\title{
The Motivations and Experiences of Young Women in a Microbicide Trial in the USA and Puerto Rico
}

\author{
Rebecca Giguere ${ }^{1}$, Gregory D. Zimet ${ }^{2}$, Jessica A. Kahn ${ }^{3}$, Curtis Dolezal ${ }^{1}$, Cheng-Shiun Leu ${ }^{1}$, \\ Marina Mabragaña ${ }^{1}$, Ian McGowan ${ }^{4}$, Alex Carballo-Diéguez ${ }^{1}$ \\ ${ }^{1}$ HIV Center for Clinical and Behavioral Studies at New York State Psychiatric Institute and Columbia University, New York, USA; \\ ${ }^{2}$ Department of Pediatrics, Indiana University School of Medicine, Indianapolis, USA; ${ }^{3}$ Cincinnati Children's Hospital Medical Cen- \\ ter and the University of Cincinnati College of Medicine, Cincinnati, USA; ${ }^{4}$ Division of Gastroenterology, Hepatology, and Nutrition, \\ Department of Medicine, University of Pittsburgh School of Medicine, Pittsburgh, USA. \\ Email: giguere@nyspi.columbia.edu
}

Received May 23 ${ }^{\text {rd }}$, 2013; revised June $23^{\text {rd }}, 2013$; accepted July $23^{\text {rd }}, 2013$

Copyright (C 2013 Rebecca Giguere et al. This is an open access article distributed under the Creative Commons Attribution License, which permits unrestricted use, distribution, and reproduction in any medium, provided the original work is properly cited.

\begin{abstract}
Young women are an important target group in microbicide research, yet little is known about why they participate and stay in microbicide trials. Our study examined motivations for participating in a Phase I microbicide trial among 61 women ages 18 - 24 years in the continental USA and Puerto Rico. We also examined their perspectives on study participation. Participants underwent a semi-structured in-depth interview in which they were asked about factors that motivated enrollment and their experiences while participating. They also completed a Web-based Computer Assisted Self Interview in which they were asked to rate study burden $(1=$ low to $4=$ high). Factors that motivated enrollment were altruism (29\%), compensation (17\%), a combination of altruism and compensation (37\%) and free medical exams (17\%). Factors that encouraged participants to stay in the study were study staff (95\%), confirmation of good health (41\%), and the opportunity to learn about their bodies (17\%). Mean ratings of study burden ranged from 1.83 (having to travel to site) to 2.41 (colposcopy), indicating that participants were not highly bothered by visits or procedures. Although Phase I trials require invasive procedures, participants were not highly bothered by them and recognized them as necessary. Good relationships with staff and clear information about how procedures contribute to study goals may encourage participants to remain in trials. Young women may be motivated to enter microbicide trials by stressing the role they will play in discovering better HIV-prevention methods and highlighting the comprehensive preventive exams they will receive.
\end{abstract}

Keywords: USA; Puerto Rico; Microbicides; Motivation; Research Participation; Young Women; HIV

\section{Introduction}

The results of the CAPRISA 004 microbicide trial in 2010 gave hope to HIV-prevention researchers that tenofovir $1 \%$ gel might reduce women's risk of contracting HIV by $39 \%$ [1]. Although a subsequent large trial [2,3] failed to confirm the results, new trials are being undertaken by other agents as researchers continue the quest for an efficacious and acceptable topical microbicide. For these trials to be successful, researchers must recruit and retain thousands of women in studies that may last up to three years. Young women between the ages of 15 and 24 years are a particularly important target group in microbicides research given that they account for $26 \%$ of new HIV infections globally [4]. In sub-Saharan Africa, they are eight times more likely than men to be HIV positive [5].
Therefore, learning how to improve recruitment and retention of young women in microbicide trials will allow researchers to understand better the safety, efficacy, and acceptability of microbicide candidates among this target population [6].

Several factors that motivate participants to enter clinical trials for HIV-prevention have been identified in prior studies: personal health benefit, [7-9] societal health benefit, [7-11] financial compensation, [7-9,12-14] trust in health professionals or researchers, [15] and support of relatives or partners for trial participation $[7,14,16]$. Conversely, barriers to recruitment and retention in clinical trials include dislike of needles, $[9,10,17]$ concerns about study burden, $[8,10]$ safety concerns $[8-11,17,18]$, and mistrust of researchers $[9,10]$. 
However, most of this literature has focused on potential trial participants rather than actual ones. Information is particularly lacking on why young women enroll in microbicide studies, especially in a Phase I safety trial that needs healthy participants with no immediate reason to test a drug whose benefits and risks are unknown. Because younger age has been associated with loss to follow-up among women in microbicide and HIV vaccine trials, $[19,20]$ knowledge of how to retain this population is important.

Our study explored factors that motivated young women between the ages of 18 and 24 to enroll and remain in a Phase I microbicide trial. We highlighted important lessons for recruitment and retention of this key population for future trials.

\section{Methods}

\subsection{Sample}

The data for this study came from a placebo-controlled Phase I study of the safety and acceptability of VivaGel ${ }^{\circledR}$ applied vaginally in sexually active young women (MTN004) and an ancillary study of microbicide-use adherence, acceptability, and attitudes among women participating in MTN-004 (ATN-062). The safety and acceptability results have been reported elsewhere [21-23]. An exploratory objective of the ancillary study was to obtain feedback on study participation from the participant's perspective, including motivation to participate and experiences as a participant. Trial participants were 61 young women randomly assigned to use either a microbicide $\left(\right.$ VivaGel $^{\circledR}$ ) or a placebo gel (HEC placebo or VivaGel ${ }^{\circledR}$ placebo) and were told that it was not known if the gel would work to protect women from getting HIV, what side effects it might cause, and whether it was safe and tolerated in all women. The research took place between August 2007 and November 2009 at three sites: Pittsburgh, Pennsylvania; San Juan, Puerto Rico; and Tampa, Florida. Participants were recruited through media advertisements, flyers, and lists of former research participants who agreed to be recontacted. Eligible study candidates were women aged 18 to 24 years who were HIV-negative, non-pregnant, sexually active, and using hormonal contraception or an intrauterine device (IUD). The study was reviewed and approved by the Institutional Review Boards at all participating institutions, and participants provided written informed consent.

\subsection{Clinical Procedures}

Participants underwent screening for eligibility prior to enrolling. Upon enrollment, they were instructed to insert an applicator with the assigned study gel in the vagina twice daily for 14 days and to use male condoms during vaginal sex. Participants returned to the clinics weekly for follow-up visits while using the study gel and again one week after completing study gel usage (final study visit), for a total of five clinic visits, spanning one month. At each visit participants gave urine and blood samples and underwent a pelvic exam (including speculum and bimanual exams) and a targeted physical exam (including vital signs and an abdominal exam). They also underwent a colposcopy at enrollment and again upon completing the study gel regimen (no biopsies were taken). Participants received approximately US\$400 for completing all clinic visits.

\subsection{Behavioral Procedures}

In addition to clinical procedures, participants agreed to complete several behavioral procedures, including three Web-based Computer-Assisted Self Interviews (CASI) in English or Spanish, two semi-structured in-depth interviews via video teleconference with a bilingual research assistant, and twice daily reporting of every occasion of product use using a telephone diary, also available in English or Spanish. Participants received up to US\$130 for completing the behavioral procedures.

\subsection{Quantitative Measures}

Demographic information was collected at baseline via CASI. At the final study visit, participants responded to a CASI about study burden, including how much they were bothered by the number and length of study visits, burden of travel to and wait time at the study site, having pelvic exams and a colposcopy, and adequacy of compensation.

\subsection{Qualitative Measures}

The qualitative interviews were conducted using a webcam for video and a telephone for the audio portion with open-ended questions and follow-up probes based on an interview guide. At the final clinic visit, the interview explored women's motivations for participating and their experiences as trial participants. The questions focused on reasons for enrolling, whether they would enroll again and under what conditions, favorite and least favorite aspects of participating, including what they thought about the type and number of procedures required, and suggestions for improving future studies.

The audio portion of the qualitative interviews was recorded and transcribed, and the transcripts were verified for accuracy by comparison with the audio recording. The final visit teleconference lasted on average $31 \mathrm{~min}-$ utes (range $=12$ - 65 minutes). Fifty-nine of the 61 women agreed to be interviewed.

\subsection{Data Analysis}

A codebook that incorporated categories and themes 
from the interview guide was developed to analyze data from qualitative interviews [24]. The codebook included definitions, inclusion and exclusion criteria, and examples of passages for inclusion. To validate and finalize the codebook, three researchers coded an initial set of three transcripts independently and then compared the codes to assess concordance. Any discrepancies were discussed until consensus was reached. The codebook was modified where necessary, and researchers coded the remaining transcripts independently using QSR NVivo 8.0 software for qualitative data analysis, [25] coding every fifth transcript in pairs to ensure intercoder agreement. Comparisons yielded over $90 \%$ agreement consistently. Coding reports were generated for the following codes: best and worst aspects of the study, motivation for participation, and suggestions for future studies. Coding reports were analyzed using content analysis, [26] then summarized and discussed by team members. So that groups could be compared, participants were then categorized based on their responses to the question: What motivated you to participate in this study? Finally, the first author selected quotes that contributed to understanding participants' motivations and experiences and, when necessary, translated them from Spanish into English.

Descriptive statistics were generated for demographic variables and study burden ratings. After four motivation categories were created based on qualitative analysis, Fisher's exact tests were used to compare the four motivation groups on categorical demographic variables (race/ ethnicity, study site, employment status, and education status). ANOVAs were used to compare the four groups on continuous variables (income and age). Analysis was performed using SPSS Statistics v.18.0 [27].

\section{Results}

\subsection{Participant Demographics}

The mean age of respondents was 21 years, and the majority of participants were White or Hispanic/Latina with some college education. Seventy percent of participants were employed either full or part-time. There were no statistically significant differences in any of the demographic characteristics among participants based on motivation for enrolling (see Table 1).

\subsection{Motivation to Enroll}

Participants were asked in the qualitative interviews, "What motivated you to enroll in this study?" Their responses fit four categories: 1) altruism; 2) the compensation; 3) medical exams; 4) multiple reasons.

Almost a third of the participants (29\%) were motivated principally by altruistic reasons. These included

Table 1. Motivations for participating by demographic characteristic $(\mathrm{N}=59)$.

\begin{tabular}{|c|c|c|c|c|}
\hline & Altruism & Compensation & Medical Exams & Multiple Reasons \\
\hline & N (\%) & $\mathbf{N}(\%)$ & $\mathbf{N}(\%)$ & $\mathbf{N}(\%)$ \\
\hline Total & $17(29 \%)$ & $10(17 \%)$ & $10(17 \%)$ & $22(37 \%)$ \\
\hline Race/Ethnicity & $\mathbf{N}(\%)$ & $\mathbf{N}(\%)$ & $\mathbf{N}(\%)$ & $\mathbf{N}(\%)$ \\
\hline White & $9(53 \%)$ & $5(50 \%)$ & $3(30 \%)$ & $11(50 \%)$ \\
\hline Hispanic/Latina & $6(35 \%)$ & $4(40 \%)$ & $7(70 \%)$ & 7 (32\%) \\
\hline Black/African-American & $1(6 \%)$ & $1(10 \%)$ & $0(0 \%)$ & $3(14 \%)$ \\
\hline Asian/Pacific-Islander & $1(6 \%)$ & $0(0 \%)$ & $0(0 \%)$ & $1(5 \%)$ \\
\hline \multicolumn{5}{|l|}{ Study Site } \\
\hline Tampa & $9(53 \%)$ & $4(40 \%)$ & $3(30 \%)$ & 13 (59\%) \\
\hline Pittsburgh & $3(17 \%)$ & $3(30 \%)$ & $1(10 \%)$ & $3(14 \%)$ \\
\hline San Juan & $5(29 \%)$ & $3(30 \%)$ & $6(60 \%)$ & $6(27 \%)$ \\
\hline \multicolumn{5}{|l|}{ Employment \& Education } \\
\hline Employed (Full or Part-Time) & $13(77 \%)$ & $7(70 \%)$ & $6(60 \%)$ & $15(68 \%)$ \\
\hline \multirow[t]{2}{*}{ In School (Full or Part-Time) } & $13(77 \%)$ & $8(80 \%)$ & $10(100 \%)$ & $20(91 \%)$ \\
\hline & M (SD) & M (SD) & M (SD) & M (SD) \\
\hline Annual Income & $\$ 8395$ (7394) & $\$ 10,840$ (6998) & $\$ 7791$ (4582) & $\$ 8479$ (6429) \\
\hline Age (in Years) & $20.53(1.70)$ & $21.00(1.83)$ & $20.60(1.51)$ & 21.00 (1.69) \\
\hline
\end{tabular}


"helping other women," "making a difference," and "contributing to a product that could be life-changing for everybody.” For example, one participant felt that being only 18 years old, "it's cool to be a part of something as big as this, that I normally wouldn't have the opportunity to be a part of." (Participant \#06) Others were happy to participate in a study they saw as "empowering women... giving us a say in having protection.” (\#53) Another participant had a more personal reason: "There are many girls who have multiple partners and don't protect themselves, and they don't realize how horrible it is to have that disease. My uncle died of AIDS, so I don't know, maybe that motivated me, too." (\#60)

Nearly one-fifth of the participants (17\%) were motivated to join primarily due to the compensation; up to approximately US\$530 for completing all study procedures. Many stated that the compensation allowed them to pay off debts or purchase items for their homes and children. As one participant stated, "Honestly, the financial incentive motivated me because it helped me a lot economically. I could pay off some debts because I was out of work for a while.” (\#25)

In addition, $17 \%$ of women mentioned the opportunity to get free medical exams as their main reason for joining the study. One woman explained, "I had a problem with my insurance plan... I couldn't get tests like a Pap smear or blood tests because the plan wanted to charge me, it was a mess. [With the study] I could have the results of the tests, and I like to always be on top of how my body is, if I am healthy, if not, what I have to do.” (\#01)

Over a third of the participants (37\%) gave multiple reasons; most stated that the compensation piqued their interest initially, and when they learned more about the study, they felt they would be contributing to something important. As one participant stated, "I guess the thing that first drew me to it was the compensation. Then when I got more involved, and I learned about what this could do for other people, I think that definitely drove me to continue. So the compensation is great, and it definitely helped in my decision to enroll, but you can't really help that other thing where you want to help other people, you want to be a part of something that can do well for others.” (\#46)

\subsection{What Participants Liked about the Study}

The retention rate for the study was $100 \%$. We asked participants about their experiences in the study to understand which aspects of participation encouraged them to remain in the study and which contributed to study burden. When asked, "What did you like most about participating in this trial?" participants often named multiple positive aspects of study participation. Almost all participants (95\%) mentioned the study staff, including the coordinators and clinicians, as a positive aspect. Participants felt comfortable with the staff, some emphasizing that they were at ease during examinations since many staff members were also young women. They also appreciated being able to ask the clinicians questions. A few participants were particularly enthusiastic, stating that they would miss seeing the study staff every week once the study ended.

Another positive aspect mentioned by $41 \%$ of participants was getting intensive, free, regular medical exams and the reassurance of finding out they were healthy. For example, one participant (\#38) stated that she was glad to get tested for sexually transmitted infections since she knew her boyfriend had had multiple partners before their relationship started.

Furthermore, $17 \%$ of women said that the colposcopy examination presented an opportunity to learn more about their bodies. As one young woman said, "At first I felt a little uncomfortable, but then it became something fascinating." (\#39) Another woman stated, "I felt it was neat because I was able to see exactly what my cervix looks like, what it does, and why. And I asked a lot of questions... so I thought that was very informational." (\#16)

Thirty-four percent of participants mentioned contributing to a good cause as the best part of participating in the study. As one participant (\#21) commented: "The best part was the cause, for sure. The only reason why I came back and continued to let people poke around and draw blood and stuff is, you know, I know that's a really good cause, and hopefully it'll get approved and really help a lot of people."

Finally, when asked about the video interviews, about half of participants (49\%) appreciated having the chance to give their opinions: "I felt like someone cared, like-I wasn't just a test subject... my opinion really mattered." (\#43) Others appreciated that this aspect of the study was easy (7\%) and used novel technology (7\%); one said that it helped her to grow more comfortable with discussing her sexuality. In addition, several participants (12\%) appreciated the extra financial compensation for the qualitative interviews.

\subsection{What Participants Disliked about the Study}

We also asked participants, "What did you like least about participating in this trial?” Again, often participants named multiple aspects of study participation that they disliked. Fifty-eight percent of participants disliked the pelvic examinations, including the colposcopy. One woman (\#10) said, "And the colpo exams. Those were hell... It just hurt really bad-the first one especially.” In addition, $14 \%$ of participants disliked the large number of staff in the room during the colposcopy (typically de- 
scribed as four or more), and one was uncomfortable with a male resident observing the pelvic exam and asking questions.

Thirty-two percent of participants stated that they disliked weekly blood draws. One commented that the amount of blood drawn was excessive: "I didn't feel like they needed that much blood. They usually have like five or six vials of blood.” (\#53) Conversely, another participant stated that she was afraid of needles but that having to get her blood drawn weekly helped her to overcome that fear and that she was willing to do it "to help others." (\#57)

A number of participants disliked the time commitment involved in participating. Thirty-one percent of participants stated that study participation often involved a long wait at the clinic, and some described study visits of up to four hours. This was especially hard for women with children or who were working and in school. A few participants mentioned excessive paperwork for participants and staff, and one found the consent process to be too long. Finally, $22 \%$ of participants mentioned that the video in-depth interviews took too long.

Despite these complaints, very few participants described being highly bothered by the procedures, and almost all mentioned they had been through many of them before or expected them as part of the study. Some also explained that knowing why the procedures were necessary helped them to withstand them. As one woman (\#59) stated, "The thing is, you need to have all the tests and everything to get things accurate-get things passed and everything. So it was just expected. Like, I knew why they needed to take my blood. Obviously I knew why they were going down in my vagina, because they definitely need to check that out-it needs to be right.” Others qualified their comments by saying that they got used to the procedures over time or taking a matter-of-fact attitude; for example, a participant (\#40) said "Of course those are bothersome exams, but I agreed to do this. It wasn't like forced upon me type of thing."

\subsection{Study Burden}

Responses to the quantitative assessment on study burden ( $1=$ Not bothered at all to $4=$ Bothered very much) confirmed qualitative findings, as the most burdensome factors included the colposcopy $(\mathrm{M}=2.41)$, the length of the study visits (2.37), and pelvic exams (2.14). Nevertheless, the mean ratings for study burden were all $<3$ out of 4 , indicating that participants were not highly bothered by study visits or procedures (see Table 2).

\subsection{Suggestions for Improvement}

Thirty-six percent of participants did not have any suggestions for improving the study. Of those who did, many focused on how to decrease the wait-time for participants while at the clinic, including reducing the paperwork that study coordinators filled out; having either two study doctors or one clinician dedicated only to the study; and streamlining the information collected from participants.

Other suggestions focused on increasing comfort and convenience such as allowing participants more flexibility in scheduling visit times; providing snacks during long visits; providing childcare for women with children; reducing the number of people in the exam room; and conducting the in-depth interview at the beginning of the visit so that participants are not tired or hungry during the interview.

A few participants (10\%) had suggestions for recruitment, including modifying the recruitment flyer to provide more information about the study and using online modes of recruitment including Facebook and MySpace. Another suggested having recruiters available in person to answer questions for prospective participants (Some of these techniques were used in this study).

Nevertheless, participants often modified their comments by acknowledging that the procedures were clearly necessary: "I think everything that they're doing here has a purpose, and it is the way it is for a reason.” (\#49). Another (\#59) said, “... even though the colposcopy is not the most comfortable thing in the world, I know it needs needs to be done, so I wouldn't change that."

\section{Discussion}

This study was the first, to our knowledge, to explore factors motivating young women to enroll and remain in

Table 2. Study burden ( $\mathrm{N}=59)$.

\begin{tabular}{cc}
\hline I was bothered by... & \\
\hline Study Visits & M (SD) \\
Number of Study Visits & $1.97(1.19)$ \\
Length of Study Visits & $2.37(0.89)$ \\
Having to Travel to Study Site & $1.83(1.19)$ \\
Waiting Time at Visits & $1.92(1.15)$ \\
Study Procedures & \\
Pelvic Exams & $2.14(1.14)$ \\
Colposcopy & $2.41(1.13)$ \\
Study compensation & $\mathbf{N}(\%)$ \\
Not Enough & $2(3 \%)$ \\
About Right & $49(83 \%)$ \\
More than Enough & $8(14 \%)$ \\
\hline
\end{tabular}

${ }^{\mathrm{a}}$ Four point scale from 1 = Strongly disagree to 4 = Strongly agree. 
a microbicide trial. The majority of participants were motivated to join the study for altruistic or financial reasons. Our findings confirm that previous research on willingness to participate in HIV-prevention trials may apply to participants in a microbicide trial $[7,10,11]$ and add to the body of literature showing that, although compensation plays a part in motivation to enroll and return for follow-up, a combination of factors that also include altruism and personal convenience is at play [28,29].

Based on our findings, an effective way to motivate young women to enter microbicide trials could be to stress the important role they will play in helping to discover better HIV-prevention methods for women. This could be especially relevant for those who, like one of our participants, know someone who is HIV-infected. Nonetheless, helping to empower other women motivated many of the young women, and most emphasized the importance of doing something for others. Some enjoyed being part of a project they considered important; hence, making women feel that they are partners with research staff to advance science may encourage participation.

Participants also frequently mentioned the free medical exams and diagnostic tests that they received as another motivating factor. In addition, young women in our study appreciated forming relationships with clinic staff and learning about sexual health. Given that young adults within the USA commonly have low rates of health insurance, [30] access to preventive care and follow-up may be an important motivating factor for some young women to participate in trials.

Our findings confirm the importance of having friendly, informative study staff. Prior research has shown that the researcher-participant relationship is critical, that friendliness and helpfulness of staff influence recruitment and retention, and that good communication is highly valued by participants [31]. Good relationships with staff that can provide clear information about how procedures contribute to study goals may encourage participants to remain in trials and to be adherent to product use. Ensuring that participants have repeated contact with the same staff members at every visit can facilitate the development of these relationships.

Finally, although Phase I trials can require invasive procedures, our participants are not highly bothered by them and recognized them as necessary. Given that participants are enthusiastic about receiving medical care and feel comfortable with study staff, these factors may have mitigated the burden of weekly invasive procedures.

Although a major strength of our study is our diverse sample of young women and rich qualitative data, our results are not generalizable given our relatively small US-based convenience sample and the specific focus of this trial on an experimental vaginal microbicide. Also, participants may have self-censored their feedback on study burden during the videoconferences out of a desire not to criticize the interviewer directly. Another limitation is that this was a one-month study and motivations might be markedly different for longer phase 2 and 3 trials in developing world settings. Finally, we did not measure barriers to enrollment among those who were screened but chose not to enroll in the study.

Our study shows that participants' motivations can be multi-faceted and may include financial need, personal health benefit, and a desire to help others. Motivations also may change over the course of the study; therefore, greater attention throughout the study to what motivates participants to return for visits and their perceived level of study burden will likely affect retention. Finally, deeply engaging participants as partners in research is essential because their perception of the importance of their role in reaching the study's goals may influence their faithfulness to the protocol and ensure the success of the study, bringing us closer to developing an efficacious microbicide.

\section{Acknowledgements}

The authors would like to thank Ana Ventuneac for her invaluable support as research project manager, Emily Maynard for her help with interviewing participants and coding data for this study, and Timothy Frasca, Mobolaji Ibitoye, and Tsitsi Masvawure for their help and guidance during the writing process. In addition, we greatly appreciate the hard work of the study staff at the sites, and are indebted to the study participants for volunteering their time and for their willingness to discuss personal matters with us. This research was sponsored by the US National Institutes of Health (NIH), and cosponsored by CONRAD and Starpharma Pty Ltd. The studies were designed and implemented by the Microbicide Trials Network (MTN-004, Ian McGowan, PI) and the Adolescent Trials Network (ATN-062, Alex Carballo-Diéguez, PI). The MTN (UM1AI068633) has been funded by NIAID, NICHD, and NIMH. MTN-004 and ATN-062 were also funded through NICHD awards to the Adolescent Trials Network (U01HD040533 and U01 HD040474) co-funded by NIMH and NIDA. The study products were provided free of charge by Starpharma Pty Ltd. The Statistical Center was supported by NIAID (U01AI068615). Additional support came from the National Institute of Mental Health to the HIV Center for Clinical and Behavioral Studies at NY State Psychiatric Institute and Columbia University (P30-MH43520; Principal Investigator: Anke A. Ehrhardt). The content is solely the responsibility of the authors and does not necessarily represent the official views of NIH.

\section{REFERENCES}

[1] Q. Abdool Karim, S. S. Abdool Karim, J. A. Frohlich, A. 
C. Grobler, C. Baxter, L. E. Mansoor, et al., "Effectiveness and Safety of Tenofovir Gel, an Antiretroviral Microbicide, for the Prevention of HIV Infection in Women,” Science, Vol. 329, No. 5996, 2010, pp. 1168-1174. doi:10.1126/science.1193748

[2] M. E. Quiñones-Mateu and G. Vanham, "HIV Microbicides: Where Are We Now?” Current HIV Research, Vol. 10, No. 1, 2012, pp. 1-2.

[3] A. van der Straten, L. Van Damme, J. E. Haberer and D. R. Bangsberg, "Unraveling the Divergent Results of PreExposure Prophylaxis Trials for HIV Prevention,” AIDS, Vol. 26, No. 7, 2012, pp. F13-F19. doi:10.1097/QAD.0b013e3283522272

[4] UNAIDS, “AIDS at 30: Nations at the Crossroads,” 2011. http://www.unaids.org/unaids_resources/aidsat30/aids-at30.pdf

[5] UNAIDS, "UNAIDS Report on the Global AIDS Epidemic 2010,” 2010. http://www.unaids.org/globalreport/global_report.htm

[6] R. J. DiClemente, M. S. Ruiz and J. M. Sales, "Barriers to Adolescents' Participation in HIV Biomedical Prevention Research,” Journal of Acquired Immune Deficiency Syndromes, Vol. 54 No. S1, 2010, pp. S12-S17. doi:10.1097/QAI.0b013e3181e1e2c0

[7] G. Colfax, S. Buchbinder, G. Vamshidar, C. Celum, D. McKirnan, J. Neidig, et al., "Motivations for Participating in an HIV Vaccine Efficacy Trial," Journal of Acquired Immune Deficiency Syndromes, Vol. 39, No. 3, 2005, pp. 359-364. doi:10.1097/01.qai.0000152039.88422.ec

[8] P. A. Newman, N. Duan, K. J. Roberts, D. Seiden, E. T. Rudy, D. Swendeman, et al., "HIV Vaccine Trial Participation among Ethnic Minority Communities: Barriers, Motivators, and Implications for Recruitment,” Journal of Acquired Immune Deficiency Syndromes, Vol. 41, No. 2, 2006, pp. 210-217. doi:10.1097/01.qai.0000179454.93443.60

[9] C. D. Voytek, K. T. Jones and D. S. Metzger, "Selectively Willing and Conditionally Able: HIV Vaccine Trial Participation among Women at 'High Risk' of HIV Infection,” Vaccine, Vol. 29, No. 36, 2011, pp. 6130-6135. doi:10.1016/j.vaccine.2011.06.064

[10] S. P. Buchbinder, B. Metch, S. E. Holte, S. Scheer, A. Coletti and E. Vittinghoff, "Determinants of Enrollment in a Preventive HIV Vaccine Trial: Hypothetical versus Actual Willingness and Barriers to Participation,” Journal of Acquired Immune Deficiency Syndromes, Vol. 36, No. 1, 2004, pp. 604-612. doi:10.1097/00126334-200405010-00009

[11] R. P. Strauss, S. Sengupta, S. Kegeles, E. McLellan, D. Metzger, S. Eyre, et al., "Willingness to Volunteer in Future Preventive HIV Vaccine Trials: Issues and Perspectives from Three US Communities," Journal of Acquired Immune Deficiency Syndromes, Vol. 26, No. 1, 2001, pp. 63-71.

[12] L. Balfour, K. Corace, G. A. Tasca, C. Tremblay, J.-P. Routy and J. B. Angel, "Altruism Motivates Participation in a Therapeutic HIV Vaccine Trial (CTN 173)," AIDS Care, Vol. 22, No. 11, 2010, pp. 1403-1409. doi:10.1080/09540121003693522
[13] R. A. Jenkins, S. Chinaworapong, P. A. Morgan, C. Ruangyuttikarn, A. Sontirat, J. Chiu, et al., "Motivation, Recruitment, and Screening of Volunteers for a Phase I/II HIV Preventive Vaccine Trial in Thailand,” Journal of Acquired Immune Deficiency Syndromes, Vol. 18, No. 2, 1998, pp. 171-177. doi:10.1097/00042560-199806010-00009

[14] K. Tharawan, C. Manopaiboon, C. Ellertson, K. Limpakarnjanarat, S. Chaikummao, P. H. Kilmarx, et al., "Women's Willingness to Participate in Microbicide Trials in Northern Thailand," Journal of Acquired Immune Deficiency Syndromes, Vol. 28, No. 2, 2001, pp. 180-186.

[15] E. R. Volkmann, D. Claiborne and J. S. Currier, "Determinants of Participation in HIV Clinical Trials: The Importance of Patients' Trust in Their Provider," HIV Clinical Trials, Vol. 10, No. 2, 2009, pp. 104-109. doi:10.1310/hct1002-104

[16] C. Woodsong, P. Alleman, P. Musara, A. Chandipwisa, M. Chirenje, F. Martinson, et al., "Preventive Misconception as a Motivation for Participation and Adherence in Microbicide Trials: Evidence from Female Participants and Male Partners in Malawi and Zimbabwe," AIDS Behavior, Vol. 16, No. 3, 2012, pp. 785-790. doi:10.1007/s10461-011-0027-7

[17] E. Ruzagira, S. Wandiembe, L. Bufumbo, J. Levin, M. A. Price, H. Grosskurth, et al., "Willingness to Participate in Preventive HIV Vaccine Trials in a Community-Based Cohort in South Western Uganda,” Tropical Medicine \& International Health, Vol. 14, No. 2, 2009, pp. 196-203. doi:10.1111/j.1365-3156.2008.02200.x

[18] J. M. O’Connell, R. S. Hogg, K. Chan, S. A. Strathdee, N. McLean, S. L. Martindale, et al., "Willingness to Participate and Enroll in a Phase 3 Preventive HIV-1 Vaccine Trial," Journal of Acquired Immune Deficiency Syndromes, Vol. 31, No. 5, 2002, pp. 521-528. doi:10.1097/00126334-200212150-00010

[19] G. deBruyn, M. G. Hudgens, P. S. Sullivan and A. C. Duerr, "Participant Retention in Clinical Trials of Candidate HIV Vaccines,” Journal of Acquired Immune Deficiency Syndromes, Vol. 39, No. 4, 2005, pp. 499-501. doi:10.1097/01.qai.0000148532.12329.df

[20] P. J. Feldblum, V. Halpern, C.-C. Lie, O. Obunge, F. Ogunsola, W. Ampofo, et al., "What Predicts Non-Retention in Microbicide Trials?” Contemporary Clinical Trials, Vol. 32, No. 4, 2011, pp. 512-516. doi:10.1016/j.cct.2011.03.008

[21] A. Carballo-Diéguez, R. Giguere, C. Dolezal, B. A. Chen, J. Kahn, G. Zimet, et al., “Tell Juliana’: Acceptability of the Candidate Microbicide VivaGel ${ }^{\circledR}$ and Two Placebo Gels among Ethnically Diverse, Sexually Active Young Women Participating in a Phase 1 Microbicide Study," AIDS Behavior, Vol. 16, No. 7, 2012, pp. 1761-1774. doi:10.1007/s10461-011-0028-6

[22] I. McGowan, K. Gomez, K. Bruder, I. Febo, B. A. Chen, B. A. Richardson, et al., "Phase 1 Randomized Trial of the Vaginal Safety and Acceptability of SPL7013 Gel (VivaGel) in Sexually Active Young Women (MTN004),” AIDS, Vol. 25, No. 8, 2011, pp. 1057-1064. doi:10.1097/QAD.0b013e328346bd3e 
[23] R. Giguere, A. Carballo-Diéguez, A. Ventuneac, M. Mabragaña, C. Dolezal, B. A. Chen, et al., "Variations in Microbicide Gel Acceptability among Young Women in the USA and Puerto Rico,” Cult Health Sexuality, Vol. 14, No. 2, 2011, pp. 151-166. doi:10.1080/13691058.2011.630099

[24] K. M. MacQueen, E. McLellan, K. Kay and B. Milstein, "Codebook Development for Team-Based Qualitative Analysis,” Cultural Anthropology Methods, Vol. 10, No. 2, 1998, pp. 31-36.

[25] "NVivo Qualitative Data Analysis Software; QSR International Pty Ltd. Version 8, 2008,” 2008.

[26] M. Q. Patton, "Qualitative Research and Evaluation Methods,” SAGE, Thousand Oaks, 2002.

[27] "PASW Statistics 18, Release Version 18.0.0 (SPSS, Inc., 2009, Chicago, IL, www.spss.com).”

[28] R. W. Grant and J. Sugarman, "Ethics in Human Subjects
Research: Do Incentives Matter?” Journal of Medicine and Philosophy, Vol. 29, No. 6, 2004, pp. 717-738. doi:10.1080/03605310490883046

[29] E. B. D. Ripley, "A Review of Paying Research Participants: It's Time to Move beyond the Ethical Debate," Journal of Empirical Research on Human Research Ethics: An International Journal, Vol. 1, No. 4, 2006, pp. 9-20. doi:10.1525/jer.2006.1.4.9

[30] S. T. Callahan and W. O. Cooper, "Uninsurance and Health Care Access among Young Adults in the United States," Pediatrics, Vol. 116, No. 1, 2005, pp. 88-95. doi:10.1542/peds.2004-1449

[31] C. Scott, J. Walker, P. White and G. Lewith, "Forging Convictions: The Effects of Active Participation in a Clinical Trial,” Social Science \& Medicine, Vol. 72, No. 12, 2011, pp. 2041-2048. doi:10.1016/j.socscimed.2011.04.021 